\title{
Multiplicities of Points on Schubert Varieties in Grassmannians
}

JOACHIM ROSENTHAL*

rosenthal.1@nd.edu

Department of Mathematics, University of Notre Dame, Notre Dame, Indiana 46556, USA

ANDREI ZELEVINSKY

andrei@neu.edu

Northeastern University, Department of Mathematics, Boston, MA 02115, USA

Received January 26, 1999

Abstract. We obtain an explicit determinantal formula for the multiplicity of any point on a classical Schubert variety.

Keywords: Schubert varieties, singularities, multiplicities, partial difference equation

\section{Main result}

An important invariant of a singular point on an algebraic variety $X$ is its multiplicity: the normalized leading coefficient of the Hilbert polynomial of the local ring. The main result of the present note is an explicit determinantal formula for the multiplicities of points on Schubert varieties in Grassmannians. This is a simplification of a formula obtained in [5]. More recently, the recurrence relations for multiplicities of points on more general (partial) flag varieties were obtained in [2,3]. However, to the best of our knowledge the case of Grassmannians remains the only case for which an explicit formula for multiplicities is available.

Fix positive integers $d$ and $n$ with $0 \leq d \leq n$, and consider the Grassmannian $G r_{d}(V)$ of $d$-dimensional subspaces in a $n$-dimensional vector space $V$ (over an algebraically closed field of arbitrary characteristic). Recall that Schubert varieties in $G r_{d}(V)$ are parameterized by the set $I_{d, n}$ of integer vectors $\mathbf{i}=\left(i_{1}, \ldots, i_{d}\right)$ such that $1 \leq i_{1}<\cdots<i_{d} \leq n$. For a given complete flag $\{0\}=V_{0} \subset V_{1} \subset \cdots \subset V_{n}=V$, the Schubert variety $X_{\mathbf{i}}$ is defined as follows:

$$
X_{\mathbf{i}}:=\left\{W \in G r_{d}(V) \mid \operatorname{dim}\left(W \bigcap V_{i_{k}}\right) \geq k \text { for } k=1, \ldots, d\right\} .
$$

The Schubert cell $X_{\mathbf{i}}^{0}$ is an open subset in $X_{\mathbf{i}}$ given by

$$
X_{\mathbf{i}}^{0}:=\left\{W \in X_{\mathbf{i}} \mid \operatorname{dim}\left(W \bigcap V_{i_{k}-1}\right)=k-1 \text { for } k=1, \ldots, d\right\} .
$$

*The authors were partially supported by NSF grants DMS-9610389 and DMS-9625511. 
It is well known that the Schubert variety $X_{\mathbf{i}}$ is the disjoint union of Schubert cells $X_{\mathbf{j}}^{0}$ for all $\mathbf{j} \leq \mathbf{i}$ in the componentwise partial order on $I_{d, n}$. The multiplicity of a point $x$ in $X_{\mathbf{i}}$ is constant on each Schubert cell $X_{\mathbf{j}}^{0} \subset X_{\mathbf{i}}$, and we denote this multiplicity by $M_{\mathbf{j}}(\mathbf{i})$.

Our main result is the following explicit formula for $M_{\mathbf{j}}(\mathbf{i})$ (where the binomial coefficients $\left(\begin{array}{l}a \\ b\end{array}\right)$ are subject to the condition that $\left(\begin{array}{c}a \\ b\end{array}\right)=0$ for $\left.b<0\right)$ :

Theorem 1 The multiplicity $M_{\mathbf{j}}(\mathbf{i})$ of a point $x \in X_{\mathbf{j}}^{0} \subset X_{\mathbf{i}}$ is given by

$$
M_{\mathbf{j}}(\mathbf{i})=(-1)^{s_{1}+\cdots+s_{d}} \operatorname{det}\left[\begin{array}{cccc}
\left(\begin{array}{c}
i_{1} \\
-s_{1}
\end{array}\right) & \ldots & \ldots & \left(\begin{array}{c}
i_{d} \\
-s_{d}
\end{array}\right) \\
\left(\begin{array}{c}
i_{1} \\
1-s_{1}
\end{array}\right) & \cdots & \cdots & \left(\begin{array}{c}
i_{d} \\
1-s_{d}
\end{array}\right) \\
\vdots & & & \vdots \\
\left(\begin{array}{c}
i_{1} \\
d-1-s_{1}
\end{array}\right) & \cdots & \cdots & \left(\begin{array}{c}
i_{d} \\
d-1-s_{1}
\end{array}\right)
\end{array}\right] \text {, }
$$

where

$$
s_{q}:=\#\left\{j_{p} \mid i_{q}<j_{p}\right\} .
$$

The proof of Theorem 1 will be given in the next section. Although determinants of matrices formed by binomial coefficients were extensively studied by combinatorialists (see, e.g., [1]), the experts whom we consulted did not recognize the determinant in (1).

We conclude this section by an example illustrating Theorem 1 .

Example 2 Assume the indices $\mathbf{i}, \mathbf{j}$ satisfy $j_{d} \leq i_{1}$. In this situation the numbers $s_{1}, \ldots, s_{d}$ attain the smallest possible value: $s_{1}=\cdots=s_{d}=0$. Then the $(p, q)$-entry of the determinant in (1) has the form $P_{p}\left(i_{q}\right)$, where $P_{p}(t)$ is a polynomial with the leading term $t^{p-1} /(p-1)$ !. It follows that

$$
M_{\mathbf{j}}(\mathbf{i})=\frac{1}{1 ! \cdots(d-1) !} V(\mathbf{i})=\frac{1}{1 ! \cdots(d-1) !} \prod_{p>q}\left(i_{p}-i_{q}\right),
$$

where $V(\mathbf{i})$ is the Vandermonde determinant $\operatorname{det}\left(\left(i_{q}^{p-1}\right)\right)$.

\section{Proof of Theorem 1}

Fix two vectors $\mathbf{j} \leq \mathbf{i}$ from $I_{d, n}$, and let

$$
\operatorname{deg}(\mathbf{j}, \mathbf{i}):=d-\#\left\{i_{q} \mid i_{q} \in\left\{j_{1}, \ldots, j_{d}\right\}\right\} .
$$

For a nonnegative integer vector $\mathbf{s}=\left(s_{1}, \ldots, s_{d}\right)$, we set

$$
|\mathbf{s}|:=s_{1}+\cdots+s_{d} .
$$


As shown in [5] and [3, page 202], the multiplicity $M_{\mathbf{j}}(\mathbf{i})$ satisfies the initial condition $M_{\mathbf{j}}(\mathbf{j})=1$ and the partial difference equation

$$
M_{\mathbf{j}}(\mathbf{i})=\frac{1}{\operatorname{deg}(\mathbf{j}, \mathbf{i})} \sum_{\mathbf{k}} M_{\mathbf{j}}(\mathbf{k}),
$$

where the sum is over all $\mathbf{k} \in I_{d, n}$ such that $\mathbf{j} \leq \mathbf{k}<\mathbf{i}$, and $|\mathbf{k}|=|\mathbf{i}|-1$.

To prove (1), we proceed by induction on $|\mathbf{i}|$. The initial step is to verify (1) for $\mathbf{i}=\mathbf{j}$. In this case the numbers $s_{1}, \ldots, s_{d}$ attain their maximum possible value: $s_{q}=d-q$. It follows that

$$
(-1)^{|\mathbf{s}|} \operatorname{det}\left[\begin{array}{cccc}
0 & \ldots & 0 & 1 \\
\vdots & & 1 & * \\
0 & \therefore & \therefore & \vdots \\
1 & * & \ldots & *
\end{array}\right]=1=M_{\mathbf{j}}(\mathbf{j}),
$$

as required.

For the inductive step, we introduce some notation. To any nonnegative integer vector $\mathbf{s}=\left(s_{1}, \ldots, s_{d}\right)$ we associate a polynomial $P_{\mathbf{s}}(\mathbf{t}) \in \mathbb{Q}[\mathbf{t}]=\mathbb{Q}\left[t_{1}, \ldots, t_{d}\right]$ defined by

$$
P_{\mathbf{s}}(\mathbf{t})=(-1)^{|\mathbf{s}|} \operatorname{det}\left[\begin{array}{cccc}
\left(\begin{array}{c}
t_{1} \\
-s_{1}
\end{array}\right) & \ldots & \ldots & \left(\begin{array}{c}
t_{d} \\
-s_{d}
\end{array}\right) \\
\left(\begin{array}{c}
t_{1} \\
1-s_{1}
\end{array}\right) & \ldots & \ldots & \left(\begin{array}{c}
t_{d} \\
1-s_{d}
\end{array}\right) \\
\vdots & & & \vdots \\
\left(\begin{array}{c}
t_{1} \\
d-1-s_{1}
\end{array}\right) & \ldots & \ldots & \left(\begin{array}{c}
d-1-s_{d} \\
d-1
\end{array}\right)
\end{array}\right]
$$

here $\left(\begin{array}{l}t \\ s\end{array}\right)$ is the polynomial $t(t-1) \cdots(t-s+1) / s$ ! for $s \geq 0$, and $\left(\begin{array}{l}t \\ s\end{array}\right)=0$ for $s<0$. Thus our goal is to show that $M_{\mathbf{j}}(\mathbf{i})=P_{\mathbf{s}}(\mathbf{i})$ with $\mathbf{s}$ given by (2).

For $q=1, \ldots, d$, let $\Delta_{q}: \mathbb{Q}[\mathbf{t}] \rightarrow \mathbb{Q}[\mathbf{t}]$ denote the partial difference operator $\Delta_{q} P(\mathbf{t})=$ $P(\mathbf{t})-P\left(\mathbf{t}-e_{q}\right)$, where $e_{1}, \ldots, e_{d}$ are the unit vectors in $\mathbb{Q}^{d}$. Here is the key lemma.

Lemma 3 For any nonnegative integer vector $\mathbf{s}$, the corresponding polynomial $P_{\mathbf{s}}(\mathbf{t})$ satisfies the partial difference equation

$$
\left(\Delta_{1}+\cdots+\Delta_{d}\right) P=0 .
$$

Proof: First notice that the Vandermonde determinant $V(\mathbf{t})=\prod_{p>q}\left(t_{p}-t_{q}\right)$ satisfies (7) since it is a non-zero skew-symmetric polynomial of minimal possible degree, and the operator $\Delta_{1}+\cdots+\Delta_{d}$ preserves the space of skew-symmetric polynomials. The vector 
space of solutions of (7) is also invariant under translations $\mathbf{t} \mapsto \mathbf{t}+\mathbf{k}$ so it is enough to show that each $P_{\mathbf{s}}(\mathbf{t})$ is a linear combination of polynomials $V(\mathbf{t}+\mathbf{k})$. Here is the desired expression:

$$
P_{\mathbf{s}}(\mathbf{t})=\frac{1}{1 ! \cdots(d-1) !} \sum_{0 \leq \mathbf{k} \leq \mathbf{s}}(-1)^{|\mathbf{k}|}\left(\begin{array}{l}
s_{1} \\
k_{1}
\end{array}\right) \cdots\left(\begin{array}{l}
s_{d} \\
k_{d}
\end{array}\right) V(\mathbf{t}+\mathbf{k}) .
$$

Let us prove (8). The same argument as in Example 2 above shows that

$$
\frac{1}{1 ! \cdots(d-1) !} V(\mathbf{t}+\mathbf{k})=\operatorname{det}\left[\begin{array}{cccc}
\left(\begin{array}{c}
t_{1}+k_{1} \\
0
\end{array}\right) & \ldots & \ldots & \left(\begin{array}{c}
t_{d}+k_{d} \\
0
\end{array}\right) \\
\left(\begin{array}{c}
t_{1}+k_{1} \\
1
\end{array}\right) & \ldots & \ldots & \left(\begin{array}{c}
t_{d}+k_{d} \\
1
\end{array}\right) \\
\vdots & & & \vdots \\
\left(\begin{array}{c}
t_{1}+k_{1} \\
d-1
\end{array}\right) & \ldots & \ldots & \left(\begin{array}{c}
t_{d}+k_{d} \\
d-1
\end{array}\right)
\end{array}\right] .
$$

Substituting this expression into (8) and performing the multiple summation, we see that the right hand side becomes the determinant of the $d \times d$ matrix whose $(p, q)$-entry is

$$
\sum_{k_{q}=0}^{s_{q}}(-1)^{k_{q}}\left(\begin{array}{l}
s_{q} \\
k_{q}
\end{array}\right)\left(\begin{array}{c}
t_{q}+k_{q} \\
p-1
\end{array}\right)=(-1)^{s_{q}}\left(\begin{array}{c}
t_{q} \\
p-1-s_{q}
\end{array}\right)
$$

(the last equality is a standard binomial identity). This completes the proof of (8) and Lemma 3.

One last piece of preparation before performing the inductive step: the Pascal binomial identity $\left(\begin{array}{l}t \\ s\end{array}\right)=\left(\begin{array}{c}t-1 \\ s\end{array}\right)+\left(\begin{array}{c}t-1 \\ s-1\end{array}\right)$ implies that

$$
\Delta_{q} P_{\mathbf{s}}(\mathbf{t})=-P_{\mathbf{s}+e_{q}}\left(\mathbf{t}-e_{q}\right)
$$

for any nonnegative integer vector $\mathbf{s}$ and any $q=1, \ldots, d$.

To conclude the proof of Theorem 1 , suppose that $\mathbf{j}<\mathbf{i}$ and assume by induction that $M_{\mathbf{j}}(\mathbf{k})$ is given by (1) for any $\mathbf{k} \in I_{d, n}$ such that $\mathbf{j} \leq \mathbf{k}<\mathbf{i}$. Let $\mathbf{s}$ be the vector given by (2). In view of (4), the desired equality $M_{\mathbf{j}}(\mathbf{i})=P_{\mathbf{s}}(\mathbf{i})$ is a consequence of the following:

$$
\operatorname{deg}(\mathbf{j}, \mathbf{i}) P_{\mathbf{s}}(\mathbf{i})-\sum_{\mathbf{k}} M_{\mathbf{j}}(\mathbf{k})=0
$$

where the sum is over all $\mathbf{k} \in I_{d, n}$ such that $\mathbf{j} \leq \mathbf{k}<\mathbf{i}$, and $|\mathbf{k}|=|\mathbf{i}|-1$.

We shall deduce (11) from the equality

$$
\sum_{q=1}^{d} \Delta_{q} P_{\mathbf{s}}(\mathbf{i})=0
$$


provided by Lemma 3 . To do this, we compute $\Delta_{q} P_{\mathbf{s}}(\mathbf{i})$ in each of the following mutually exclusive cases (we use the conventions $i_{0}=0$ and $s_{0}=d$ ):

Case $1 \quad i_{q} \notin\left\{j_{1}, \ldots, j_{d}\right\}, i_{q}-1>i_{q-1}$. Then $\mathbf{k}:=\mathbf{i}-e_{q}$ belongs to $I_{d, n}$, and we have $\mathbf{j} \leq \mathbf{k}$. Replacing $\mathbf{i}$ by $\mathbf{k}$ in (2) does not change the vector $\mathbf{s}$. By our inductive assumption, $P_{\mathbf{s}}(\mathbf{k})=M_{\mathbf{j}}(\mathbf{k})$, and so $\Delta_{q} P_{\mathbf{s}}(\mathbf{i})=P_{\mathbf{s}}(\mathbf{i})-M_{\mathbf{j}}(\mathbf{k})$.

Case $2 i_{q} \notin\left\{j_{1}, \ldots, j_{d}\right\}, i_{q}-1=i_{q-1}$. For such $q$, we have $P_{\mathbf{s}}\left(\mathbf{i}-e_{q}\right)=0$ since the corresponding determinant has the $(q-1)$ th and $q$ th columns equal to each other. Thus $\Delta_{q} P_{\mathrm{s}}(\mathbf{i})=P_{\mathrm{s}}(\mathbf{i})$.

Case $3 i_{q} \in\left\{j_{q+1}, \ldots, j_{d}\right\}, i_{q}-1>i_{q-1}$. As in Case 1 , we have $\mathbf{k}:=\mathbf{i}-e_{q} \in I_{d, n}$, and $\mathbf{j} \leq \mathbf{k}$. However now replacing $\mathbf{i}$ by $\mathbf{k}$ in (2) changes $\mathbf{s}$ to $\mathbf{s}+e_{q}$. Combining the inductive assumption with (10), we conclude that $\Delta_{q} P_{\mathbf{s}}(\mathbf{i})=-P_{\mathbf{s}+e_{q}}(\mathbf{k})=-M_{\mathbf{j}}(\mathbf{k})$.

Case $4 i_{q} \in\left\{j_{q+1}, \ldots, j_{d}\right\}, i_{q}-1=i_{q-1}$. In this case, the $d \times d$ matrix whose determinant is $P_{\mathrm{s}+e_{q}}\left(\mathbf{i}-e_{q}\right)$ has the $(q-1)$ th and $q$ th columns equal to each other, hence $\Delta_{q} P_{\mathbf{s}}(\mathbf{i})=-P_{\mathrm{s}+e_{q}}(\mathbf{k})=0$.

Case $5 i_{q}=j_{q}$. Then we have

$$
s_{1} \geq s_{2} \geq \cdots \geq s_{q-1} \geq s_{q}+1=d+1-q,
$$

and so the $d \times d$ matrix whose determinant is $P_{\mathbf{s}+e_{q}}\left(\mathbf{i}-e_{q}\right)$ has a zero $(d+1-q) \times q$ submatrix. As in Case 4, this implies $\Delta_{q} P_{\mathbf{s}}(\mathbf{i})=-P_{\mathbf{s}+e_{q}}(\mathbf{k})=0$.

Adding up the contributions $\Delta_{q} P_{\mathbf{s}}(\mathbf{i})$ from all these cases, we obtain (11); this completes the proof of Theorem 1 .

Remark 4 In [5], the multiplicity $M_{\mathbf{j}}(\mathbf{i})$ was expressed as a multiple sum given by (8).

Remark 5 The multiplicity $M_{\mathbf{j}}(\mathbf{i})$ is by definition a positive integer. The partial difference equation (4) (combined with the initial condition $M_{\mathbf{j}}(\mathbf{j})=1$ ) makes the positivity of $M_{\mathbf{j}}(\mathbf{i})$ obvious but the fact that $M_{\mathbf{j}}(\mathbf{i})$ is an integer becomes rather mysterious. On the other hand, Theorem 1 makes it clear that $M_{\mathbf{j}}(\mathbf{i})$ is an integer but not that $M_{\mathbf{j}}(\mathbf{i})>0$. It would be interesting to find an expression for $M_{\mathbf{j}}(\mathbf{i})$ that makes obvious both properties.

Remark 6 The space of all polynomial solutions of the partial difference equation (7) can be described as follows. Let $\mathbf{y}=\left(y_{1}, \ldots, y_{d}\right)$ be an auxiliary set of variables, and let $\varphi: \mathbb{Q}[\mathbf{y}] \rightarrow \mathbb{Q}[\mathbf{t}]$ be the isomorphism of vectors spaces that sends each monomial $\prod_{q=1}^{d} y_{q}^{n_{q}}$ to $\prod_{q=1}^{d} t_{q}\left(t_{q}+1\right) \cdots\left(t_{q}+n_{q}-1\right)$. The map $\varphi$ intertwines each $\Delta_{q}$ with the partial derivative $\frac{\partial}{\partial y_{q}}$. It follows that the space of solutions of (7) is the image under $\varphi$ of the $\mathbb{Q}$-subalgebra in $\mathbb{Q}[\mathbf{y}]$ generated by all differences $y_{p}-y_{q}$. 
Remark 7 In the special case when $\mathbf{j}=(1,2, \ldots, d)$, the following determinantal formula for the multiplicity $M_{\mathbf{j}}$ (i) was given in [3]. Let $\lambda$ be the partition $\left(i_{d}-d, \ldots, i_{2}-\right.$ $\left.2, i_{1}-1\right)$, and let $\lambda=\left(\alpha_{1}, \ldots, \alpha_{r} \mid \beta_{1}, \ldots, \beta_{r}\right)$ be the Frobenius notation of $\lambda$ (see [4]). According to [3], $M_{\mathbf{j}}(\mathbf{i})$ is equal to the determinant of the $r \times r$ matrix whose $(p, q)$-entry is $\left(\alpha_{p}+\beta_{q}\right)$. It is not immediately clear why this determinantal expression agrees with the one given by (1).

\section{Acknowledgments}

We are grateful to V. Lakshmibai who initiated this project by suggesting to one of us (J. R.) to publish the results of his thesis [5]. We thank Sergey Fomin, Ira Gessel and Jerzy Weyman for helpful conversations.

Added in proof: the questions raised in Remarks 5 and 7 have been resolved by C. Krattenthaler in his preprint "On multiplicities of points on Schubert varieties in Grassmannians," arXiv: math. AG/0011129, November, 2000.

\section{References}

1. I. Gessel and G.X. Viennot, "Binomial determinants, paths, and hooklength formulae," Adv. Math. 58 (1985), 300-321.

2. V. Lakshmibai, "Multiplicities of points on a Schubert variety," C. R. Acad. Sci. Paris Sér. I Math. 321(2), (1995), 215-218.

3. V. Lakshmibai and J. Weyman, "Multiplicities of points on a Schubert variety in a minuscule G/P," Adv. Math. 84 (1990), 179-208

4. I.G. Macdonald, Symmetric Functions and Hall Polynomials, 2nd edition, Clarendon Press, Oxford, 1995.

5. J. Rosenthal, "Schubertvarietäten und deren Singularitäten,” Diplom Thesis, University of Basel, Switzerland, 1986. 